University of Wollongong

Research Online

Faculty of Engineering - Papers (Archive)

Faculty of Engineering and Information

Sciences

May 2004

\title{
Virgin magnetization of a magnetically shielded superconductor wire: Theory and experiment
}

\author{
Y. A. Genenko \\ Technische Universitat Darmstadt, Germany \\ S. V. Yampolskii \\ Technische Universitat Darmstadt, Germany \\ Alexey Pan \\ University of Wollongong, pan@uow.edu.au
}

Follow this and additional works at: https://ro.uow.edu.au/engpapers

Part of the Engineering Commons

https://ro.uow.edu.au/engpapers/137

\section{Recommended Citation}

Genenko, Y. A.; Yampolskii, S. V.; and Pan, Alexey: Virgin magnetization of a magnetically shielded superconductor wire: Theory and experiment 2004.

https://ro.uow.edu.au/engpapers/137

Research Online is the open access institutional repository for the University of Wollongong. For further information contact the UOW Library: research-pubs@uow.edu.au 


\title{
Virgin magnetization of a magnetically shielded superconductor wire: Theory and experiment
}

\author{
Yu. A. Genenko a) and S. V. Yampolskiiib) \\ Institut für Materialwissenschaft, Technische Universität Darmstadt, D-64287 Darmstadt, Germany
}

\author{
A. V. Pan \\ Institute for Superconducting and Electronic Materials, University of Wollongong, Wollongong, \\ NSW 2522, Australia
}

(Received 19 January 2004; accepted 18 March 2004; published online 29 April 2004)

\begin{abstract}
On the basis of exact solutions to the London equation, the magnetic moment of a type II superconductor filament surrounded by a soft-magnet environment is calculated and the procedure of extracting the superconductor contribution from magnetic measurements is suggested. A comparison of theoretical results with experiments on $\mathrm{MgB}_{2} / \mathrm{Fe}$ wires allows the estimation of the value of critical current for the first magnetic flux penetration. (c) 2004 American Institute of Physics. [DOI: 10.1063/1.1741036]
\end{abstract}

Recently hybrid systems composed of superconducting and soft-magnetic materials attracted much attention in view of possibilities to improve superconductor performance by shielding out an external field as well as a transport current self-field. ${ }^{1-3}$ Very intense investigations were carried out on superconducting $\mathrm{MgB}_{2}$ wires sheathed in iron, which became ideal objects to explore the magnetic shielding effect due to the simplicity of their fabrication. As was observed in recent experiments, such structures exhibit enhanced superconducting critical currents over a wide range of the external magnetic field. ${ }^{4,5}$

The commonly used technique for the estimation of the critical current value is the measurement of the superconductor magnetization versus applied magnetic field. Usually such measurements are carried out as follows. ${ }^{5,6}$ The total magnetization of $\mathrm{MgB}_{2} / \mathrm{Fe}$ wire is measured in the superconducting state (below $T_{c}$ ) and in the normal state (above $T_{c}$ ). After that the magnetization of superconducting core is determined by subtraction of the latter results from the former ones (because in the normal state, only the magnetic sheath is magnetized). The magnetization of the superconductor allows one to estimate the critical current value which is proportional to the height of the hysteretic magnetic loop. It is assumed in this procedure that the magnetization of iron sheath does not depend on the presence of the superconductor and, hence, is identical above and below $T_{c}$. However, it is intuitively clear that this assumption may be somewhat incorrect. Indeed, due to the Meissner effect below $T_{c}$, the superconductor expels the magnetic flux into the sheath. This expulsion does not happen in the normal state where the magnetic field is homogeneous in the cylindrical magnetically shielded cavity. ${ }^{7}$ Therefore, the magnetic field distribution in the magnet sheath as well as its magnetization can be different, depending on whether the core is in the superconducting state or in the normal one. Recently, this scenario has been supported by the magneto-optical visualization of local

\footnotetext{
${ }^{\text {a)} E l e c t r o n i c ~ a d d r e s s: ~ y u g e n e n @ t g m . t u-d a r m s t a d t . d e ~}$

${ }^{b)}$ On leave from: Donetsk Institute for Physics and Technology, National Academy of Sciences of Ukraine, 83114 Donetsk, Ukraine.
}

flux distributions within the iron sheath of a $\mathrm{MgB}_{2}$ superconducting wire. $^{8}$

In the present letter, we calculate exactly the distribution of magnetic field inside and outside a superconducting filament sheathed by a magnet layer, as well as the magnetization of such a structure in the region of reversible magnetic behavior, i.e., for the flux-free (Meissner) state of the superconductor and well below the saturation field of the magnet. Comparing theoretical results with experiment, we verify the above described procedure of the superconducting critical current estimation.

Let us consider an infinite cylindrical superconductor filament of radius $R$ enveloped in a coaxial cylindrical magnetic sheath of thickness $d$ with relative permeability $\mu$ and exposed to the external magnetic field $\mathbf{H}_{0}$ perpendicular to the cylinder axis (Fig. 1).

We start from the London equation for the magnetic induction $\mathbf{B}_{S C}$ in the superconducting area ${ }^{9}$

$$
\mathbf{B}_{S C}+\lambda^{2} \text { curl curl } \mathbf{B}_{S C}=0,
$$

with the London penetration depth $\lambda$. The field outside the superconductor denoted by $\mathbf{H}_{M}$ in a magnetic sheath and by $\mathbf{H}_{\text {out }}$ in a surrounding free space is described by the Maxwell equations

$$
\text { curl } \mathbf{H}=0, \quad \operatorname{div} \mathbf{H}=0,
$$

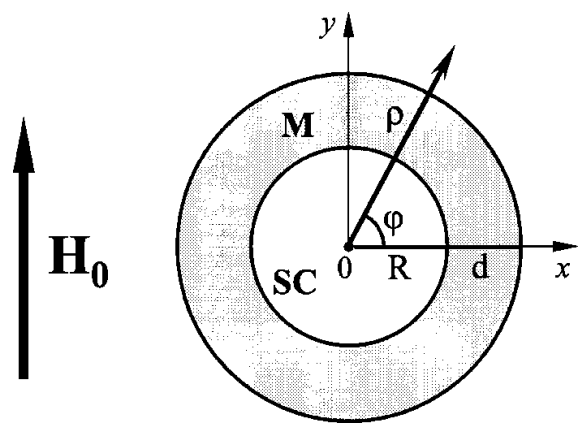

FIG. 1. Cross-sectional view of a superconductor filament covered by a coaxial cylindrical magnetic sheath. 
the latter of which is valid in the whole space. Implying an insulating nonmagnetic layer of thickness much less than $d$ and $R$ between the superconductor and the magnet sheath, ${ }^{10}$ the boundary conditions read

$$
\begin{aligned}
& B_{n, S C}=\mu_{0} \mu H_{n, M}, \quad B_{t, S C}=\mu_{0} H_{t, M} ; \\
& \mu H_{n, M}=H_{n, \text { out }}, \quad H_{t, M}=H_{t, \text { out }},
\end{aligned}
$$

for the normal $(n)$ and tangential $(t)$ components on the superconductor/magnet interface [Eq. (3a)] and on the outer magnet surface [Eq. (3b)], respectively. In addition, the field $\mathbf{H}_{\text {out }}$ has to asymptotically approach the external field $\mathbf{H}_{0}$. In cylindrical coordinates, $(\rho, \varphi, z)$ coaxial with the filament the solution of Eqs. (1) and (2) is

$$
\begin{aligned}
& B_{\rho, S C}=\mu_{0} H_{0} A_{S C}\left[I_{0}(\rho / \lambda)-I_{2}(\rho / \lambda)\right] \sin \varphi, \\
& B_{\varphi, S C}=\mu_{0} H_{0} A_{S C}\left[I_{0}(\rho / \lambda)+I_{2}(\rho / \lambda)\right] \cos \varphi,
\end{aligned}
$$

in the superconductor;

$$
\begin{aligned}
& H_{\rho, M}=H_{0}\left(A_{M 1}-A_{M 2} R^{2} / \rho^{2}\right) \sin \varphi, \\
& H_{\varphi, M}=H_{0}\left(A_{M 1}+A_{M 2} R^{2} / \rho^{2}\right) \cos \varphi,
\end{aligned}
$$

in the magnet sheath; and

$$
\begin{aligned}
& H_{\rho, \text { out }}=H_{0}\left[1+A_{\text {out }}(R+d)^{2} / \rho^{2}\right] \sin \varphi, \\
& H_{\varphi, \text { out }}=H_{0}\left[1-A_{\text {out }}(R+d)^{2} / \rho^{2}\right] \cos \varphi,
\end{aligned}
$$

in the space around the filament. The coefficients $A_{S C}, A_{M 1}$, $A_{M 2}$, and $A_{\text {out }}$ are given by

$$
\begin{aligned}
A_{S C}= & 4 \mu / \Delta, \\
A_{M 1}= & 2\left[(\mu-1) I_{0}(R / \lambda)+(\mu-1) I_{2}(R / \lambda)\right] / \Delta, \\
A_{M 2}= & 2\left[(\mu-1) I_{0}(R / \lambda)+(\mu+1) I_{2}(R / \lambda)\right] / \Delta, \\
A_{\text {out }}= & \left\{\left[(\mu-1)^{2}-(\mu+1)^{2} R^{2} /(R+d)^{2}\right] I_{2}(R / \lambda)\right. \\
& \left.+\left(\mu^{2}-1\right)\left[1-R^{2} /(R+d)^{2}\right] I_{0}(R / \lambda)\right\} / \Delta,
\end{aligned}
$$

where

$$
\begin{aligned}
\Delta= & {\left[(\mu+1)^{2}-(\mu-1)^{2} R^{2} /(R+d)^{2}\right] I_{0}(R / \lambda) } \\
& +\left(\mu^{2}-1\right)\left[1-R^{2} /(R+d)^{2}\right] I_{2}(R / \lambda) .
\end{aligned}
$$

The Meissner current density in the superconductor only has the $z$ component which equals

$$
j_{z}(\rho, \varphi)=A_{\mathrm{SC}} \frac{2 H_{0}}{\lambda} I_{1}(\rho / \lambda) \cos \varphi .
$$

A limiting case of the hollow magnetic cylinder may be obtained from Eqs. (4)-(8) by setting $\lambda \rightarrow \infty$ which results in a nonzero-homogeneous field inside the hole as expected from Ref. 7.

Now, we can easily calculate the mean magnetization of both the superconducting core and iron sheath which, due to the geometry of the problem, only has a $y$ component. The magnetization of the superconductor is

$$
M_{S C}=\frac{1}{V_{S C}} \int_{V_{S C}} d V[\boldsymbol{\rho} \times \mathbf{j}]_{y}=-2 A_{\mathrm{SC}} H_{0} I_{2}(R / \lambda),
$$

where the factor 2 is to account for the far ends of the sample. ${ }^{11}$ The magnetization of the iron sheath is

Downloaded 15 Aug 2006 to 130.130.37.6. Redistribution subject to

$$
M_{M}=\frac{\mu-1}{V_{M}} \int_{V_{M}} d V H_{y, M}=(\mu-1) H_{0} A_{M 1} .
$$

In the practically interesting case, $R \gg \lambda$, they become

$$
\begin{aligned}
& M_{S C} \simeq-\frac{4 H_{0}}{\mu+1-(\mu-1) /(1+d / R)^{2}}, \\
& M_{M} \simeq \frac{2(\mu-1) H_{0}}{\mu+1-(\mu-1) /(1+d / R)^{2}} .
\end{aligned}
$$

Although the magnetization of the sheath (13) does not contain $\lambda$, it does not coincide with the magnetization of a hollow magnetic cylinder which can be obtained from Eq. (11) by setting $\lambda \rightarrow \infty$ :

$$
M_{H C} \simeq \frac{2\left(\mu^{2}-1\right) H_{0}}{(\mu+1)^{2}-(\mu-1)^{2} /(1+d / R)^{2}} .
$$

For the ratio of these two quantities an inequality,

$$
\frac{M_{M}}{M_{H C}}=\frac{(\mu+1)^{2}-(\mu-1)^{2} /(1+d / R)^{2}}{(\mu+1)^{2}-\left(\mu^{2}-1\right) /(1+d / R)^{2}}>1,
$$

holds which means the magnetic flux density increase due to the flux expelled from the superconductor.

Although in the critical state of superconductors only part of the magnetic flux is expelled, the same inequality $M_{M}>M_{H C}$ should still be valid. Therefore, we conclude that in previous considerations, ${ }^{4-6}$ the magnetization of the sheath below $T_{c}$ could be underestimated and, hence, the magnetization of a superconductor together with the critical current value could be underestimated too.

Note that slopes of both magnetizations (13) and (14), $M_{M(H C)} / H_{0}$, have finite values at any fixed parameter $d / R$ even in the limit of $\mu \rightarrow \infty$. It differs from the case of the field parallel to the filament axis, when the shielding effect is absent for an infinitely long cylinder sheath ${ }^{7}$ and the corresponding slope $M_{M(H C)} / H_{0}=\mu-1$ rises unbounded with $\mu$. In fact, the shielding effect of a much smaller amplitude was also observed in the longitudinal geometry due to the finite length of samples. ${ }^{4,10}$

Now we use the theoretical expressions to fit experimental results. Details of the sample preparation and measurement technique were given in Ref. 5. The sample had the length of $L=4.1 \mathrm{~mm}$, the radius of the superconducting core $R=0.5 \mathrm{~mm}$, the magnet sheath thickness $d=0.25 \mathrm{~mm}$, and the permeability $\mu=46$ measured in the longitudinal field. ${ }^{10}$ The difference between magnetic moments measured in the normal and in the superconducting states was quite small (about $1 \%$ ) at fields $B_{0}=\mu_{0} H_{0}<0.1 \mathrm{~T}$ (see Fig. 2). The magnetic moment in the normal state was fitted using $M_{H C}$ (14) while the total moment below $T_{c}$ was fitted using $M_{S C}$ (12) and $M_{M}$ (13) with the only fitting parameter of ratio $\eta$ between the effective lengths of magnetic sheath and superconductor. The best fit was achieved for $\eta=1.5$. The deviation of $\eta$ from 1 indicates the significant role of the sample edge effects leading to the discrepancy between the measured magnetic moment of the relatively short sample and the moment calculated for an infinitely long cylinder with Eqs. (12)-(14).

Using the above parameters, we calculate the $M_{M}\left(B_{0}\right)$ dependence in the superconducting state from Eq. (13) which AlP license or copyright, see http://apl.aip.org/apl/copyright.jsp 


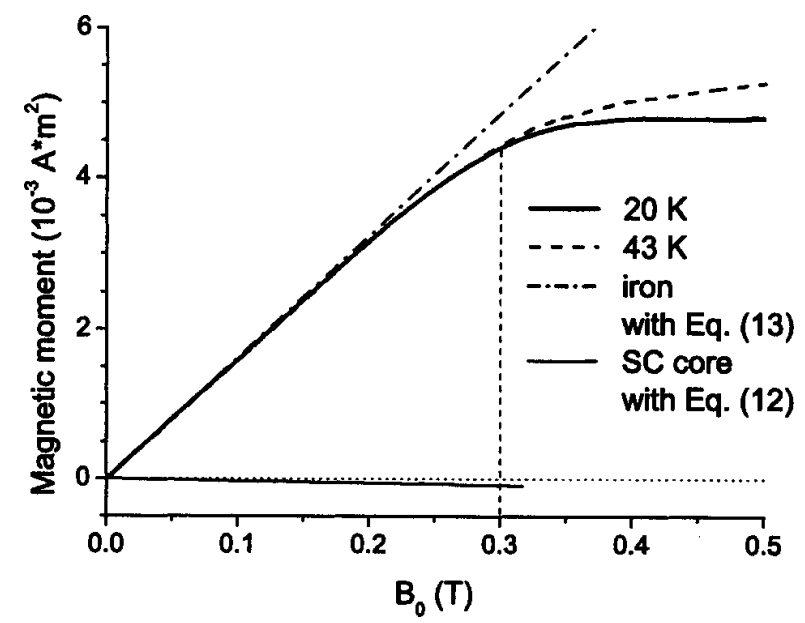

FIG. 2. The magnetic moment dependence of the $\mathrm{MgB}_{2} / \mathrm{Fe}$ wire on the magnetic field applied transversely in the superconducting state (thick solid line) and in the normal state (dashed line). The moment of the iron sheath in the superconducting state calculated using Eq. (13) is presented by the dashed-dotted line. The thin solid line exhibits the superconductor core contribution.

is shown in Fig. 2 by the dashed-dotted line. From Eqs. (13) and (14), it follows that the magnetization of the iron sheath at $T<T_{c}$ is about $3 \%$ larger than that at $T>T_{c}$. Let us note that this difference may reach $100 \%$ at smaller values of the parameters $d / R$ and $\mu$.

The response of the magnetic sheath is intrinsically nonlinear such that is clearly visible in the normal state magnetization curve at $B_{0}>0.15 \mathrm{~T}$. The deviation of the total magnetic moment below $T_{c}$ at $B_{p} \simeq 0.3 \mathrm{~T}$ from that in the normal state may be attributed to the nonlinearity due to the first magnetic flux entry into the superconductor. From the $B_{p}$ value, a critical current of the first vortex penetration may be estimated as follows.

First, from the magnetization of the superconducting core, the maximum value of screening current $j_{s}=j_{z}(R, 0)$ can be found. Combining Eqs. (9) and (10), we obtain in the case $R \gg \lambda$

$$
j_{S} \simeq 2\left|M_{S C}\right| / \lambda
$$

with $M_{S C}$ from Eq. (12). Taking $\mu=46, d / R=0.5$, and $\lambda$
$=1400 \AA$ for $T=30 \mathrm{~K}$ from Ref. 12, we obtain for $H_{0}$ $=B_{p} / \mu_{0}$ the value of $j_{s} \simeq 5 \times 10^{7} \mathrm{~A} / \mathrm{cm}^{2}$. A practically important quantity is the average density of the screening current that may be defined as $j_{c}=2 J_{c} / \pi R^{2}$, where $J_{c}$ is determined by the integration of expression (9) over one-half of the superconductor cross section. In the limit of $R \gg \lambda$, we obtain

$$
j_{c} \simeq 8\left|M_{S C}\right| / \pi R
$$

and for the parameters used we found $j_{c} \simeq 1.8 \times 10^{4} \mathrm{~A} / \mathrm{cm}^{2}$ which is in a good agreement with the results of Refs. 4, 5, and 10 .

In conclusion, we have developed a procedure of extracting the superconducting response from the low-field magnetic measurements on the iron sheathed superconductor filaments taking into account the difference between the magnetization of the magnet sheath below and above $T_{c}$.

Discussions of boundary conditions with A. Gurevich are gratefully acknowledged. This study was supported by the Deutsche Forschungsgemeinschaft (for one of the authors-S.V.Y.) and by the Australian Research Counsil (for another author-A.V.P.).

${ }^{1}$ M. Majoros, B. A. Glowacki, and A. M. Campbell, Physica C 334, 129 (2000); 338, 251 (2000).

${ }^{2}$ Y. A. Genenko, A. Usoskin, and H. C. Freyhardt, Phys. Rev. Lett. 83, 3045 (1999); Y. A. Genenko, A. Snezhko, and H. C. Freyhardt, Phys. Rev. B 62, 3453 (2000).

${ }^{3}$ H. Jarzina, C. Jooss, and H. C. Freyhardt, J. Appl. Phys. 91, 3775 (2002). ${ }^{4}$ J. Horvat, X. L. Wang, S. Soltanian, and S. X. Dou, Appl. Phys. Lett. 80, 829 (2002)

${ }^{5}$ A. V. Pan, S. H. Zhou, H. K. Liu, and S. X. Dou, Semicond. Sci. Technol. 16, L33 (2003).

${ }^{6}$ M. D. Sumption, E. W. Collins, E. Lee, X. L. Wang, S. Soltanian, and S.X. Dou, Physica C 378, 894 (2002).

${ }^{7}$ J. D. Jackson, Classical Electrodynamics (Wiley, New York, 1975).

${ }^{8}$ A. V. Pan, S. X. Dou, and T. H. Johansen, in Magneto-Optical Imaging, NATO Science series, edited by T. H. Johansen and D. V. Shantsev (Kluwer, to be published).

${ }^{9}$ P. G. de Gennes, Superconductivity of Metals and Alloys (AddisonWesley, New York, 1994).

${ }^{10}$ A. V. Pan and S. X. Dou cond-mat/0403454

${ }^{11}$ E. H. Brandt and M. Indenbom, Phys. Rev. B 48, 12893 (1993).

${ }^{12}$ P. C. Canfield, S. L. Bud'ko, and D. K. Finnemore, Physica C 385, 1 (2003). 